\title{
Numerically quantifying energy loss caused by squirt flow
}

\author{
Beatriz Quintal ${ }^{1 *}$, Eva Caspari ${ }^{1}$, Klaus Holliger ${ }^{1,2}$ and Holger Steeb ${ }^{3}$ \\ ${ }^{1}$ University of Lausanne, Lausanne, Switzerland, ${ }^{2}$ Zhejiang University, Hangzhou, China, and ${ }^{3}$ University of Stuttgart, Stuttgart, Germany
}

Received September 2018, revision accepted June 2019

\begin{abstract}
In interconnected microcracks, or in microcracks connected to spherical pores, the deformation associated with the passage of mechanical waves can induce fluid flow parallel to the crack walls, which is known as squirt flow. This phenomenon can also occur at larger scales in hydraulically interconnected mesoscopic cracks or fractures. The associated viscous friction causes the waves to experience attenuation and velocity dispersion. We present a simple hydromechanical numerical scheme, based on the interface-coupled Lamé-Navier and Navier-Stokes equations, to simulate squirt flow in the frequency domain. The linearized, quasi-static Navier-Stokes equations describe the laminar flow of a compressible viscous fluid in conduits embedded in a linear elastic solid background described by the quasi-static Lamé-Navier equations. Assuming that the heterogeneous model behaves effectively like a homogeneous viscoelastic medium at a larger spatial scale, the resulting attenuation and stiffness modulus dispersion are computed from spatial averages of the complexvalued, frequency-dependent stress and strain fields. An energy-based approach is implemented to calculate the local contributions to attenuation that, when integrated over the entire model, yield results that are identical to those based on the viscoelastic assumption. In addition to thus validating this assumption, the energy-based approach allows for analyses of the spatial dissipation patterns in squirt flow models. We perform simulations for a series of numerical models to illustrate the viability and versatility of the proposed method. For a 3D model consisting of a spherical crack embedded in a solid background, the characteristic frequency of the resulting P-wave attenuation agrees with that of a corresponding analytical solution, indicating that the dissipative viscous flow problem is appropriately handled in our numerical solution of the linearized, quasi-static Navier-Stokes equations. For 2D models containing either interconnected cracks or cracks connected to a circular pore, the results are compared with those based on Biot's poroelastic equations of consolidation, which are solved through an equivalent approach. Overall, our numerical simulations and the associated analyses demonstrate the suitability of the coupled Lamé-Navier and Navier-Stokes equations and of Biot's equations for quantifying attenuation and dispersion for a range of squirt flow scenarios. These analyses also allow for delineating numerical and physical limitations associated with each set of equations.
\end{abstract}

Key words: Attenuation, Rock physics, Squirt flow.

*E-mail: beatriz.quintal@unil.ch

\section{INTRODUCTION}

Part of the energy of mechanical waves can be converted into heat in a process that is called intrinsic attenuation, which is 
here simply referred to as attenuation. In the Earth's crust, rocks are commonly porous and saturated with water, occasionally in combination with more viscous liquids and/or gas. The presence of viscous fluids in the pores can be a major cause of wave energy dissipation through physical processes involving their displacement in the porous medium, necessarily accompanied by dissipative friction. Indeed, strong and frequency-dependent attenuation has been observed in fluidsaturated rocks, mostly through laboratory experiments, and has been interpreted as a result of wave-induced fluid pressure diffusion (FPD), often denoted wave-induced fluid flow, at the microscopic or mesoscopic scales (e.g. Best, McCann and Sothcott 1994; Sams et al. 1997; Batzle, Han and Hofmann 2006; Adelinet et al. 2010; Tisato and Quintal 2013; Pimienta, Fortin and Guéguen 2015; Subramaniyan et al. 2015; Chapman et al. 2016, 2019).

FPD at the microscopic scale is commonly referred to as squirt flow. Simple models for squirt flow are based either on interconnected flat pores which are highly compliant (O'Connell and Budiansky 1977) or on connections between these flat pores and much less compliant spherical pores (Murphy, Winkler and Kleinberg 1986). Examples of highly compliant flat pores include microcracks and grain contacts, while non-flat intergranular pores are typical examples of stiffer pores. The impact of squirt flow on attenuation from the ultrasonic to seismic frequency ranges is relatively well understood for simple distributions of the idealized pore geometries mentioned above (e.g. Mavko and Jizba 1991; Dvorkin, Mavko and Nur 1995; Chapman, Zatsepin and Crampin 2002; Gurevich et al. 2010; Collet and Gurevich 2016). In general though, studies on more realistic models of the rock microstructure are still unavailable, mainly due to the lack of appropriate numerical techniques and/or the lack of spatial resolution in microtomographic images of rock samples. From another standpoint, squirt flow is not limited to the microscopic scale, but may also occur in the mesoscopic scale range, that is, at scales larger than the pore size but smaller than the prevailing wavelength, in hydraulically interconnected mesoscopic cracks or fractures parallel to their walls (Rubino et al. 2013).

In addition to FPD associated with squirt flow at the microscopic and mesoscopic scales, significant attenuation can be caused by mesoscopic FPD associated with (i) patchy saturation, that is, heterogeneities in the pore fluid saturation due to the presence of fluids having extremely different compressibilities (White 1975; Tisato and Quintal 2013) or (ii) strong heterogeneities of the solid frame properties, such as compliant fractures embedded in a much stiffer porous background (White, Mikhaylova and Lyakhovitskiy 1975; Brajanovski,
Gurevich and Schoenberg 2005). The second phenomenon plainly differs from squirt flow in interconnected fractures as the predominant dissipative FPD takes place in the embedding porous background with the dominant direction generally away or towards the fracture. As long as the embedding background is porous and permeable, this dissipation process prevails whether the fractures are interconnected or not and, if they are, squirt flow tends to occur as well, albeit at considerably higher frequencies (Rubino et al. 2013; Quintal et al. 2014).

A series of computationally efficient methodologies to calculate attenuation due to mesoscopic FPD have been proposed in the last decade (Masson and Pride 2007; Rubino, Ravazzoli and Santos 2009; Wenzlau et al. 2010; Quintal et al. 2011). They are based on solving Biot's dynamic (1962) or quasi-static (1941) poroelastic equations through the simulation of quasi-static compression or shear tests on models that describe the representative elementary volume of a heterogeneous fluid-saturated porous medium. Based on this approach, Rubino et al. (2013) and Quintal et al. (2014) numerically study squirt flow in interconnected mesoscopic fractures, which are represented as highly compliant features of very high porosity and permeability embedded in a much stiffer background of much lower porosity and permeability. Vinci, Renner and Steeb (2014) use a hybrid-dimensional approach, where the embedding background is also described as a poroelastic medium based on Biot's (1941) equations, while the interconnected fractures are filled with a compressible fluid described by a $1 \mathrm{D}$ solution for laminar flow. A limitation of these techniques for studying classical microscopic squirt flow is that Biot's equations are conceived for the mesoscopic scale and hence it is not clear whether and to what extent they are appropriate for assessing the relevant phenomena at the microscopic scale (Burridge and Keller 1981; Whitaker 1986; Berryman 2005).

Zhang and Toksöz (2012) introduce a numerical approach for simulating squirt flow that overcomes any limitation with respect to the microscopic scale by solving a set of equations in which the elastic equation for the solid domain is coupled with the linearized Navier-Stokes equation for the fluid domain. They solve the dynamic equations, but perform quasi-static tests in the time domain, to quantify attenuation caused by squirt flow in a 3D model derived from a microtomographic image of Berea sandstone. However, only the large pores are resolved in the image, which leads them to implement a hybrid method to compensate for the cracks lost in the imaging process by combining the computational approach with a theoretical model for the crack 
distribution. More recently, microtomographic imaging with considerably larger spatial resolution has become available (Madonna et al. 2013), and important developments in the segmentation of corresponding images have been reported (Madonna, Almqvist and Saenger 2012). Combined with appropriate and efficient numerical schemes, these new techniques can be used to study squirt flow in realistic models of the rock microstructure.

Quintal et al. (2016) present a numerical approach based on the simulation of quasi-static stress relaxation tests in the time domain, which is similar to that of Zhang and Toksöz (2012), but they neglect the inertia contribution in the coupled equations. In these stress relaxation tests, a strain boundary condition is instantaneously applied to the numerical model and kept constant for a finite interval of time (e.g. Masson and Pride 2007; Quintal et al. 2011). Quintal et al. (2016) compare the results for squirt flow with those based on a numerical solution of Biot's (1941) quasi-static equations of poroelasticity considering highly porous and permeable cracks embedded in a background of very low porosity and permeability, such that attenuation and dispersion associated with FPD in the background occur at frequencies that are out of the investigated frequency range. The results of the proposed approach and of the approach based on Biot's equations show good agreement for attenuation and dispersion caused by squirt flow in interconnected cracks. Additionally, expected spatial discrepancies in the fluid velocity and dissipation fields, calculated with the two numerical schemes, are observed and discussed.

In this study, we extend and complement the study of Quintal et al. (2016). We solve the same coupled quasi-static Lamé-Navier and Navier-Stokes (LNS) equations, but here in the frequency domain and by simulating oscillatory tests. This readily allows for accurate attenuation and dispersion results in a frequency range that is broader than that for timedomain studies, in addition to greatly simplifying the numerical scheme by eliminating, together with the time derivatives, problems associated with the temporal discretization. We successfully compare results from 3D simulations with an analytical prediction for the characteristic frequency of the squirt flow dissipation phenomenon. Then, we compare 2D numerical results for attenuation and dispersion with those from an equivalent numerical scheme based on Biot's (1941) quasistatic poroelasticity considering two squirt flow scenarios: one featuring interconnected cracks and the other consisting of cracks connected to a stiffer circular pore. These comparisons allow for illustrating the physical limitations of the approach based on Biot's equations to model squirt flow and for evaluating the numerical limitations of the presented approach based on the coupled LNS equations.

\section{COUPLED LAMÉ-NAVIER AND NAVIER-STOKES EQUATIONS}

\section{Mathematical formulation}

The coupled Lamé-Navier and Navier-Stokes (LNS) equations can be used to describe laminar flow of a viscous compressible fluid in conduits embedded in an isotropic elastic, linear solid background. The set of coupled equations consists of the conservation of momentum and the constitutive equations. The conservation of momentum is given by

$\operatorname{div}(\boldsymbol{\sigma})=0$,

where $\sigma$ is the total stress tensor and div denotes its divergent. Before writing the constitutive equations, we separate the total stress tensor into its contributions in the solid and fluid phases (subscripts $s$ and $f$, respectively),

$\boldsymbol{\sigma}=\varphi \boldsymbol{\sigma}_{s}+(\varphi-1) \boldsymbol{\sigma}_{f}$

which is the basic coupling idea, where $\varphi=1$ in the solid domain, while in the fluid domain $\varphi=0$. The constitutive equations for the corresponding total stress contributions in equation (2) can be written as

$\sigma_{s}=2 \mu \operatorname{dev}(\boldsymbol{\varepsilon})+K_{s} e \mathbf{I}$,

$\boldsymbol{\sigma}_{f}=2 \eta \operatorname{dev}(\mathrm{i} \omega \boldsymbol{\varepsilon})+K_{f} e \mathbf{I}$,

where $\boldsymbol{\varepsilon}$ is the strain tensor, $\operatorname{dev}(\boldsymbol{\varepsilon})$ denotes the deviatoric strain related to the shape change, $e$ is the trace of the strain tensor related to the volume change, $\mathrm{I}$ is the identity tensor, $\mathrm{i}$ represents the imaginary unit and $\omega$ is the angular frequency. The material properties are represented by $\mu$ and $K_{s}$ denoting the shear and bulk moduli of the solid material, respectively, $K_{f}$ denoting the bulk modulus of the fluid, and $\eta$ denoting the shear viscosity of the fluid. The generalized constitutive equation is written as

$\boldsymbol{\sigma}=\varphi\left[2 \mu \operatorname{dev}(\boldsymbol{\varepsilon})+K_{s} e \mathbf{I}\right]+(\varphi-1)\left[2 \eta \operatorname{dev}(\mathrm{i} \omega \boldsymbol{\varepsilon})+K_{f} e \mathbf{I}\right]$,

and the generalized mathematical formulation, given by equations (1) and (5), can be used to describe a heterogeneous medium that has a subdomain characterizing a solid frame $(\varphi=1)$ and another corresponding to fluid-filled pores $(\varphi=$ $0)$. The components of the stress and strain tensors $\sigma$ and $\boldsymbol{\varepsilon}$ are complex valued and frequency dependent. 
In the subdomain where $\varphi=1$, equations (1) and (5) reduce to the quasi-static Lamé-Navier equations of linear elasticity and, in the subdomain where $\varphi=0$, they reduce to the linearized, quasi-static Navier-Stokes equations (Landau and Lifshitz 1959) for the laminar flow of a compressible fluid. The energy loss in this medium is entirely caused by FPD, since we use a quasi-static formulation, which means that inertial terms are absent.

Equations (1) and (5) are essentially identical to those presented by Quintal et al. (2016), except that equation (5) is written in tensor form and in the frequency domain, and the solid and fluid contributions are here explicitly separated and governed by the parameter $\varphi$.

\section{Finite-element solution}

Equations (1) and (5) are solved throughout the entire computational domain composed of spatial subdomains consisting of a solid phase $(\varphi=1)$ or a fluid phase $(\varphi=0)$. Complex solid and fluid displacements $\boldsymbol{u}(\boldsymbol{x}, \omega)$ at the boundaries between subdomains are naturally coupled, as they are described by the same unknown and are thus continuous across these boundaries. The numerical solutions for 2D and 3D problems are obtained using a finite-element direct solver and employing unstructured meshes with Delauney triangular and tetrahedral elements, respectively (Shewchuk 2002). The elements employ second-order (quadratic) shape functions with nodal points appearing in the middle of the edges of elements in addition to their vertices. This results in six nodal points for the triangular elements and ten nodal points for the tetrahedral elements. Furthermore, the material boundaries coincide exactly with element boundaries. An efficient discretization of subdomains having large aspect ratios, such as cracks, is obtained by strongly varying the sizes of the elements by a few orders of magnitude (Quintal et al. 2014, 2016). Our 2D numerical problem is equivalent to a $3 \mathrm{D}$ case under plain strain conditions, that is, no strain outside the modelling plane is allowed to develop.

To assess $\mathrm{P}$-wave attenuation and modulus dispersion, we perform an oscillatory test by applying a harmonic downward-oriented displacement homogeneously at the top boundary of the model (e.g. Rubino et al. 2009; Carcione, Santos and Picotti 2011; Milani et al. 2016). At the bottom and along the lateral boundaries of the model, the displacements in the vertical and horizontal directions, respectively, are set to zero. The S-wave attenuation can be evaluated in a similar manner by changing the boundary conditions to those of a simple-shear test.

\section{Viscoelastic approach to quantify attenuation}

Based on the assumption that the entire heterogeneous model behaves effectively as a homogeneous viscoelastic medium at a larger spatial scale, we can calculate the effective attenuation and dispersion using volume averages of the frequencydependent stress and strain fields (Jänicke, Quintal and Steeb 2015). Considering the medium as approximately isotropic, the boundary conditions described in the previous subsection allow for the calculation of the complex-valued and frequency-dependent P-wave modulus $H$ corresponding to a wave propagating in the vertical direction. The ratio between its imaginary and real parts is used to quantify the P-wave attenuation, expressed as the inverse of the quality factor (O’Connell and Budiansky 1978)

$\frac{1}{Q(\omega)}=\frac{\operatorname{Im}\{H\}}{\operatorname{Re}\{H\}}$.

\section{Energy-based approach to quantify local attenuation}

To quantify the local contribution to the total attenuation in a 2D model of a rock sample based on the coupled LNS equations, we use an energy-based approach, which is analogous to that described by Solazzi et al. (2016) for Biot's equations. The local contribution $1 / q_{k l}$ from each finite-element $\Omega_{k l}$ of the $2 \mathrm{D}$ model domain to the total attenuation $1 / Q$ is given as

$\frac{1}{Q(\omega)}=\sum_{k=1}^{n_{x}} \sum_{l=1}^{n_{y}} \frac{1}{q_{k l}(\omega)} \delta^{2}$,

where $\delta^{2}$ is the area of the element $\Omega_{k l}$. We use the definition of the inverse quality factor that corresponds exactly to the relation given in equation (6) for a linear viscoelastic material that can be modelled by a network of springs and dashpots (O’Connell and Budiansky 1978)

$\frac{1}{Q(\omega)}=\frac{\langle\Delta P(\omega)\rangle}{2 \omega\langle W(\omega)\rangle}$,

where $\langle\Delta P\rangle$ is the average power dissipated per cycle in harmonic loading and $\langle W\rangle$ is the average strain energy per cycle in the whole model domain. For our medium described by the coupled LNS equations (equations (1) and (5)), their contributions for each element $\Omega_{k l}$ of the 2D model domain can be written as functions of frequency as (e.g. Winter 1987; Jaeger, Cook and Zimmerman 2007; Plawsky 2014) 


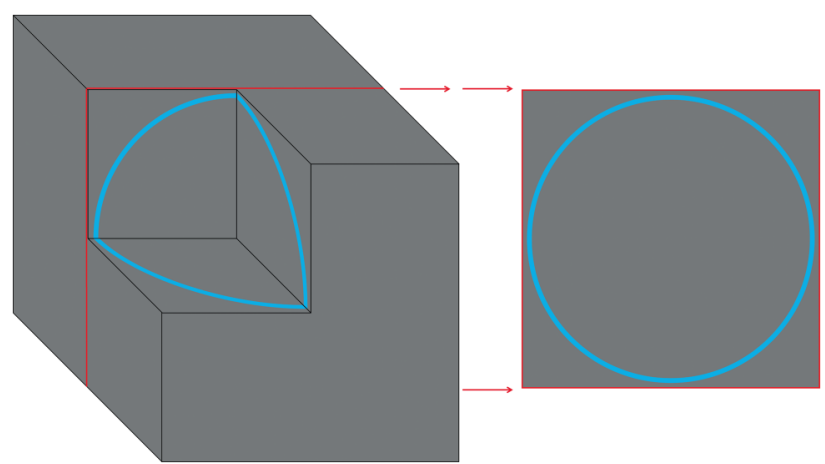

Figure 1 Sketch of a 3D numerical model embedding a spherical shell of thickness $1 \mu \mathrm{m}$ that represents a micro-crack. The side length of the cube is $150 \mu \mathrm{m}$. The embedding background (grey regions) corresponds to quartz, while the crack is filled with water (blue region). A $2 \mathrm{D}$ model consisting of a cross-section through the middle of the cubic model is shown on the right-hand side.

$$
\begin{aligned}
\left\langle\Delta P_{k l}(\omega)\right\rangle= & 2 \eta \operatorname{Re}\left\{\dot{\varepsilon}_{x x} \dot{\varepsilon}_{x x}^{*}+\dot{\varepsilon}_{y y} \dot{\varepsilon}_{y y}^{*}+\dot{\varepsilon}_{x y} \dot{\varepsilon}_{x y}^{*}\right. \\
& \left.-\frac{1}{3}\left(\dot{\varepsilon}_{x x}+\dot{\varepsilon}_{y y}\right)\left(\dot{\varepsilon}_{x x}+\dot{\varepsilon}_{y y}\right)^{*}\right\}_{k l} \delta^{2},
\end{aligned}
$$

$\left\langle W_{k l}(\omega)\right\rangle=\frac{1}{4} \operatorname{Re}\left\{\sigma_{x x} \varepsilon_{x x}^{*}+\sigma_{y y} \varepsilon_{y y}^{*}+\sigma_{x y} \varepsilon_{x y}^{*}\right\}_{k l} \delta^{2}$,

where a dot on top of a variable denotes the multiplication of the variable by $\mathrm{i} \omega$ (e.g. $\dot{\varepsilon}_{x x}=\omega \mathrm{i} \varepsilon_{x x}$ ), and the symbol * denotes the complex conjugate. Using these equations, we can quantify the local contribution $1 / q_{k l}$ from each element $\Omega_{k l}$ per unit area to the total attenuation $1 / Q$ as

$\frac{1}{q_{k l}(\omega)}=\frac{\left\langle\Delta P_{k l}(\omega)\right\rangle / \delta^{2}}{2 \omega\langle W(\omega)\rangle}$,

where the average strain energy per cycle $\langle W\rangle$ in the whole model domain is obtained by simply summing the contributions $\left\langle W_{k l}\right\rangle$ from each element $\Omega_{k l}$ throughout the entire domain

$\langle W(\omega)\rangle=\sum_{k=1}^{n_{x}} \sum_{l=1}^{n_{y}}\left\langle W_{k l}(\omega)\right\rangle$.

\section{COMPARISON WITH ANALYTICAL SOLUTION}

To compare numerical results based on the coupled LNS equations with a corresponding analytical solution, we consider a 3D numerical model containing a spherical crack embedded in an elastic solid background with the properties of quartz (Fig. 1). The subdomain within the spherical crack is described as a viscous fluid with the material properties of water. The
Table 1 Material properties for the model shown in Fig. 1

\begin{tabular}{ll}
\hline Material Property & Value \\
\hline Shear modulus of the solid $\mu$ & $44.3 \mathrm{GPa}$ \\
Bulk modulus of the solid $K_{s}$ & $37.8 \mathrm{GPa}$ \\
Shear viscosity of the fluid $\eta$ & $0.003 \mathrm{~Pa} \times \mathrm{s}$ \\
Bulk modulus of the fluid $K_{f}$ & $2.4 \mathrm{GPa}$ \\
\hline
\end{tabular}

material properties are given in Table 1 . The side of the cubic model is $150 \mu \mathrm{m}$, while the crack aperture is $1 \mu \mathrm{m}$.

The numerical results are based on the viscoelastic equivalent approach, which uses the volume averages of the frequency-dependent stress and strain fields (Fig. 2). The vertical harmonic oscillatory compression, which is applied as a displacement boundary condition on top of the model, deforms the sub-horizontal top and bottom parts of the spherical crack much more than the sub-vertical parts on the sides. This induces a fluid pressure difference between these regions, which in turn causes fluid flow, or fluid pressure diffusion

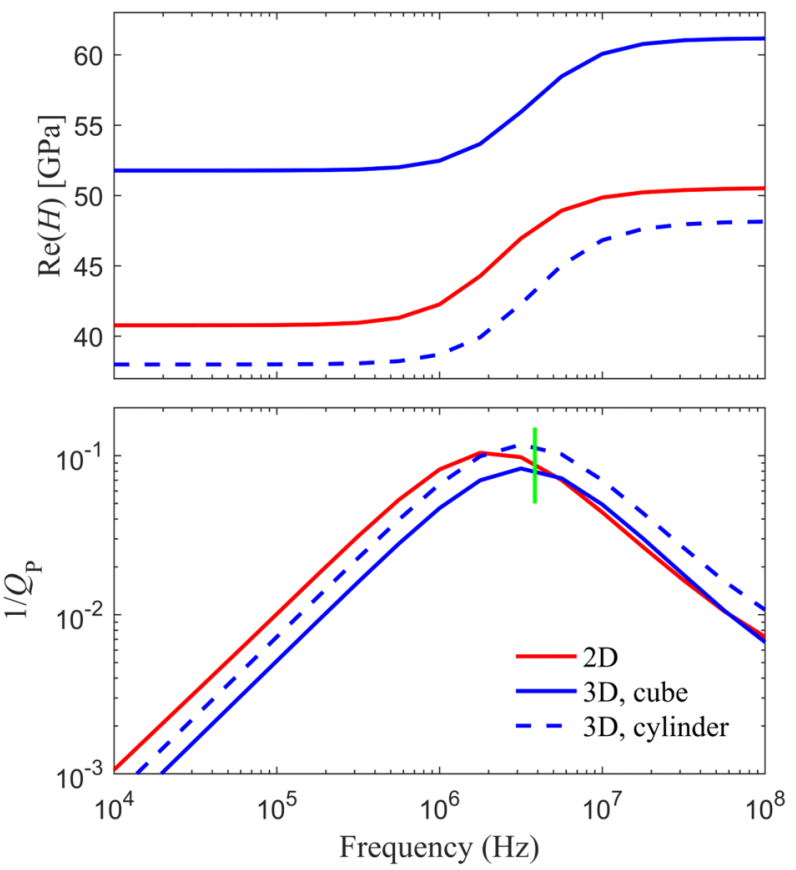

Figure 2 Real part of the P-wave modulus $\operatorname{Re}(H)$ and attenuation $1 / Q$ calculated using the viscoelastic equivalent approach from the numerical solution based on the coupled LNS equations for the 3D model shown in Fig. 1, a 3D model consisting of a cylinder embedding the spherical crack, and a 2D model consisting of a cross-section in the middle of the cubic or of the cylindrical model. The vertical green line indicates the characteristic frequency according with the analytical solution by O'Connell and Budiansky (1977) for such a spherical crack. 
(FPD), from the top and bottom parts towards the lateral part of the spherical crack and, thus, P-wave attenuation and dispersion of the P-wave modulus.

O'Connell and Budiansky (1977) analytically derived the characteristic frequency for a thin spherical shell of fluid which completely surrounds a solid sphere and is submitted to an external macroscopic shear stress field

$f_{c}=\frac{1}{2} \frac{K_{s}}{\eta} \alpha^{3}$

where $\alpha$ is the crack aspect ratio. For the special geometry considered in this squirt flow model, the crack aspect ratio $\alpha$ is expressed as the ratio of crack aperture to one quarter of the circumference of the spherical fluid shell. Using the material properties considered for our numerical model (Table 1; Fig. 1), we obtain from equation (13) the value denoted by a vertical green line in Fig. 2. The excellent agreement between this result and the characteristic frequency of the numerical results for the $3 \mathrm{D}$ cubic model shown in Fig. 1 corroborates the validity of our numerical implementation of the linearized, quasi-static Navier-Stokes equations in the framework of the coupled LNS equations.

For comparison with the numerical results for the cubic model (Fig. 1), we additionally consider (i) a 2D model consisting of a cross-section in the middle of the cubic model, as indicated on the right-hand side of Fig. 1, and (ii) a 3D cylindrical model containing an identical spherical crack with both the diameter and height of the cylinder equal to the side length of the cubic model. We use again the properties given in Table 1. The corresponding attenuation and dispersion results are also shown in Fig. 2.

Comparing results from the two 3D models, cubic and cylindrical, we observe that they have the same characteristic frequency, as expected, while the attenuation has a higher overall magnitude for the cylindrical model (Fig. 2). This occurs because the cylindrical model has a lower relative volume of elastic solid embedding the spherical crack, compared with the cubic model. This renders the cylindrical model softer, as evidenced by the generally lower real-valued P-wave modulus, which allows for a larger deformation of the spherical crack and thus results in higher energy dissipation.

Comparing the results from the cubic model containing a spherical shell with the 2D model depicted on the right-hand side of Fig. 1, we observe that the latter is characterized by a higher compressibility, higher attenuation and a shift of the characteristic frequency to a lower value (Fig. 2). These differences are consequences of the inherently larger relative crack volume of the $2 \mathrm{D}$ model. To visualize this, we can imagine replacing such a $2 \mathrm{D}$ model with a numerically equivalent $3 \mathrm{D}$ model that consists simply of an extrusion of the 2D model into a cube containing a horizontal cylindrical fluid shell. The crack volume in this $2.5 \mathrm{D}$ model is evidently larger than that in the 3D model containing a spherical crack. The aspect ratios of the two cracks are identical, as they are the ratio of crack aperture to one quarter of the circumferences of the corresponding sphere or circle, which are equal. Despite the identical aspect ratio, the different shape and volume of the crack in the $2.5 \mathrm{D}$ model allows for a larger amount of fluid to be displaced and a longer pressure equilibration process, which translates into a higher dissipation and a lower characteristic frequency, respectively. Additionally, the larger crack volume makes the 2.5D model more compressible as evidenced by a generally lower real-valued P-wave modulus, which also contributes to the increase in attenuation. The comparison between numerical results from a 3D cubic model with those of a 2D model points to the importance of $3 \mathrm{D}$ effects that are neglected in studies based on 2D models, as shown by Hunziker et al. (2018). An important aspect is that 2D models overestimate attenuation.

\section{COMPARISONS WITH SOLUTIONS OF BIOT'S EQUATIONS}

\section{Interconnected rectangular cracks}

To compare the results of our numerical scheme based on the coupled Lamé-Navier and Navier-Stokes (LNS) equations with those of an equivalent numerical scheme based on the solution of Biot's (1941) quasi-static equations (Quintal et al. 2011), we consider a 2D model, which is identical to that considered by Quintal et al. (2016) and corresponds to a fundamental block of periodically distributed

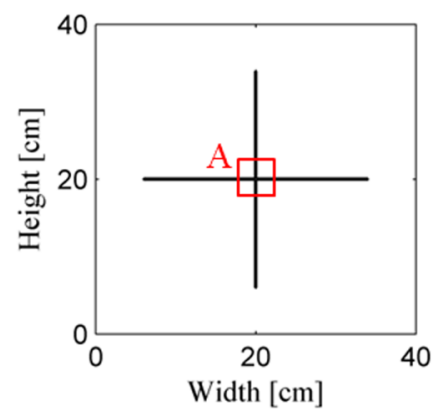

Figure 3 Sketch of the numerical model containing interconnected rectangular cracks. The aperture and length of the cracks are $1 \mathrm{~mm}$ and $14 \mathrm{~cm}$, respectively. The crack length is defined from the crack intersection to its end. 
Table 2 Material properties for the scheme based on Biot's equations

\begin{tabular}{lll}
\hline Material Parameter & Background & Crack \\
\hline Porosity & 0.06 & 0.90 \\
Permeability & $1 \mathrm{mD}$ & $8 \times 10^{7} \mathrm{mD}(=k)$ \\
Bulk modulus of the solid grain & $40 \mathrm{GPa}$ & $40 \mathrm{GPa}$ \\
Bulk modulus of the dry frame & $36 \mathrm{GPa}$ & $0.001 \mathrm{GPa}$ \\
Shear modulus of the dry frame & $44 \mathrm{Gpa}$ & $0.001 \mathrm{GPa}$ \\
Bulk modulus of the fluid & $2.4 \mathrm{GPa}$ & $2.4 \mathrm{GPa}$ \\
Shear viscosity of the fluid & $0.003 \mathrm{~Pa} \times \mathrm{s}$ & $0.003 \mathrm{~Pa} \times \mathrm{s}$ \\
\hline
\end{tabular}

Table 3 Material properties for the scheme based on the coupled LNS equations

\begin{tabular}{ll}
\hline Material Property & Value \\
\hline Shear modulus of the solid $\mu$ & $44 \mathrm{GPa}\left(=\mu_{u}\right)$ \\
Bulk modulus of the solid $K_{s}$ & $36.4 \mathrm{GPa}\left(=K_{u}\right)$ \\
Shear viscosity of the fluid $\eta$ & $0.003 \mathrm{~Pa} \times \mathrm{s}$ \\
Bulk modulus of the fluid $K_{f}$ & $2.4 \mathrm{GPa}$ \\
\hline
\end{tabular}

pairs of interconnected cracks (Fig. 3). The scheme based on Biot's (1941) quasi-static equations also accounts only for attenuation caused by FPD, by neglecting the attenuation associated with inertial effects, and is here also modified to perform simulations in the frequency domain. The two numerical schemes use identical meshes.

In order to obtain comparable results from the two numerical schemes, the material properties for the scheme based on Biot's equations are chosen to describe a solid frame having very low porosity in the embedding background and very high porosity and compressibility within the cracks (Table 2). The permeability in the background is set to a very low value. Contrastingly, since the flow direction in the cracks is essentially parallel to the crack walls, we use a solution of Stokes' equations, known as cubic law, for laminar flow between two smooth and parallel plates (e.g., Jaeger et al. 2007) to calculate the much higher permeability in the cracks

$k=\frac{h^{2}}{12}$,

where $h$ is the crack aperture. The resulting permeability for an aperture of $0.1 \mathrm{~cm}$ (Fig. 3) is shown in Table 2 together with the properties of the saturating pore fluid, which are those of water both in the cracks and in the porous background. As compression applied to open cracks will be predominantly supported by the fluid (Pride, Berryman and Harris 2004), the cracks considered in this study were simply characterized by a bulk modulus of the dry frame that is much lower than that of the saturating fluid. The shear modulus of the dry frame was chosen to have a correspondingly low value (Nakagawa and Schoenberg 2007).

A much simpler set of material properties is used for the scheme based on the coupled LNS equations and given in Table 3. The properties of the fluid within the crack are identical to those used for Biot's equations (Table 2). To allow for results that are comparable with those based on Biot's

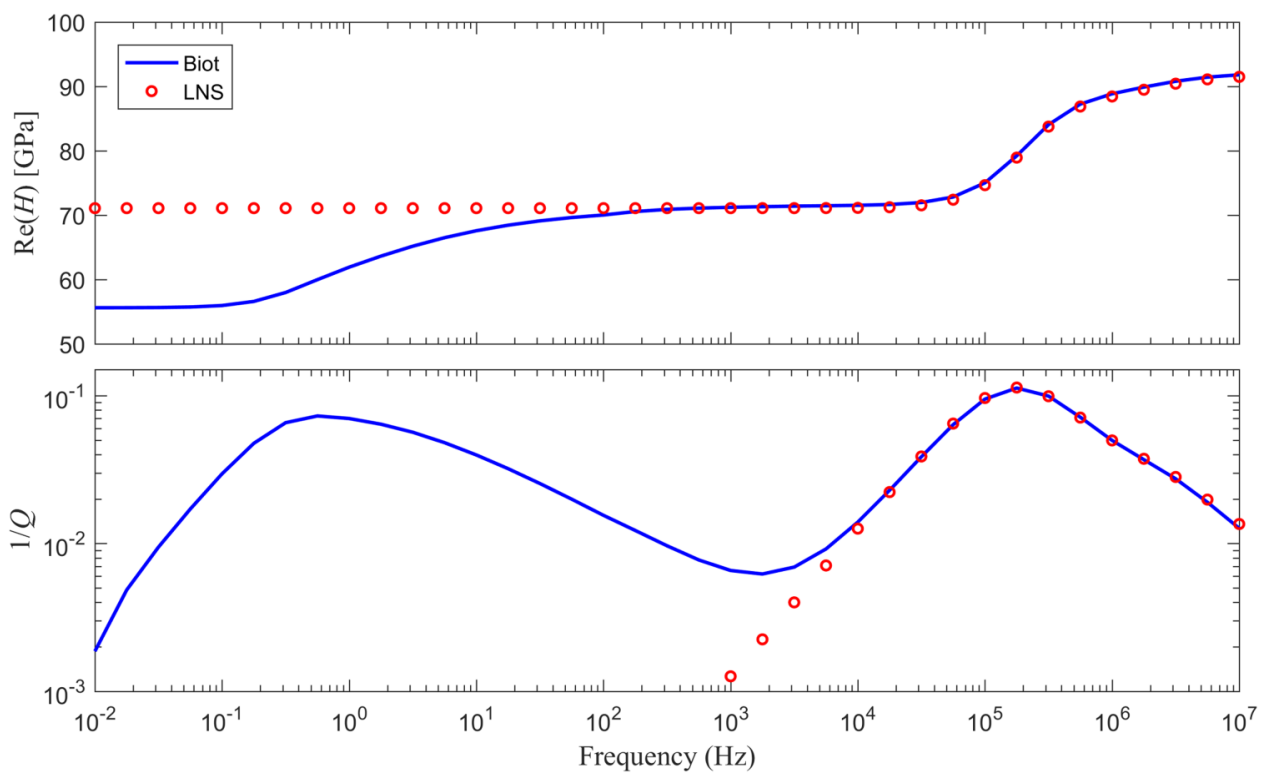

Figure 4 Real part of the P-wave modulus $\operatorname{Re}(H)$ and attenuation $1 / Q$ calculated using the viscoelastic equivalent approach from the numerical solutions based on Biot's quasi-static equations and the coupled LNS equations. 


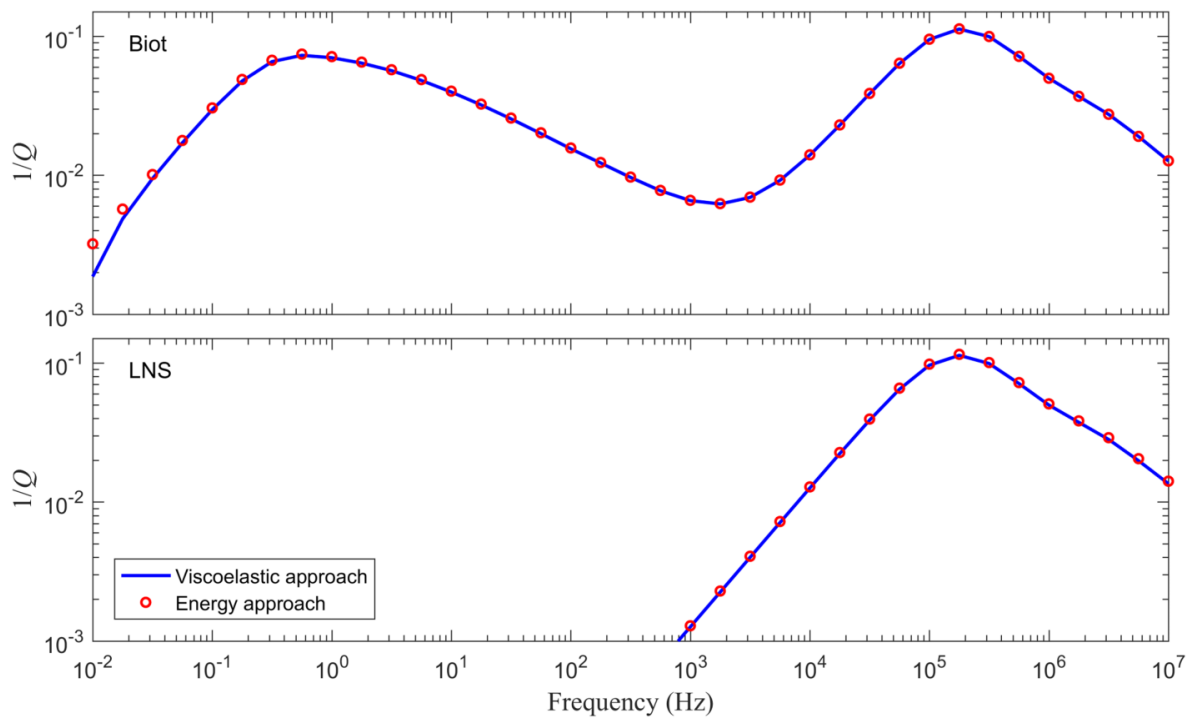

Figure 5 Attenuation $1 / Q$ calculated using the viscoelastic equivalent approach and the energy approach from the numerical solutions based on Biot's quasi-static equations and the coupled LNS equations.

equations, the bulk and shear moduli of the non-porous solid background, described by the coupled LNS equations, are set to be equal to the undrained bulk and shear moduli $K_{u}$ and $\mu_{u}$ of the poroelastic material in the porous background (Gassmann 1951).

The resulting P-wave modulus dispersion and attenuation based on both Biot's equations and on the coupled LNS equations are obtained using the viscoelastic equivalent approach (Fig. 4). For the results based on Biot's equations, we observe two attenuation peaks accompanied by two corresponding inflections of the dispersion curves. The attenuation peak and corresponding dispersion at lower frequencies are due to FPD in the background. In the compression cycle of the imposed oscillations, the fluid in the compressible cracks experiences a larger pressure increase than in the less compressible background. The resulting fluid pressure difference is the cause of FPD in the background. This low-frequency phenomenon is captured only by the scheme based on Biot's poroelastic equations because the scheme based on the coupled LNS equations describes the background embedding the cracks as an elastic non-porous solid. The attenuation peak and corresponding dispersion at higher frequencies are due to squirt flow or, more precisely, due to FPD within the interconnected cracks, parallel to the crack walls. Basically, fluid flows from one crack into the other one. This happens because, in the compression cycle of the vertically imposed oscillations, only the horizontal cracks are significantly deformed. Thus, fluid in the horizontal cracks experiences a larger pressure increase than in the less deformed vertical crack. The resulting fluid
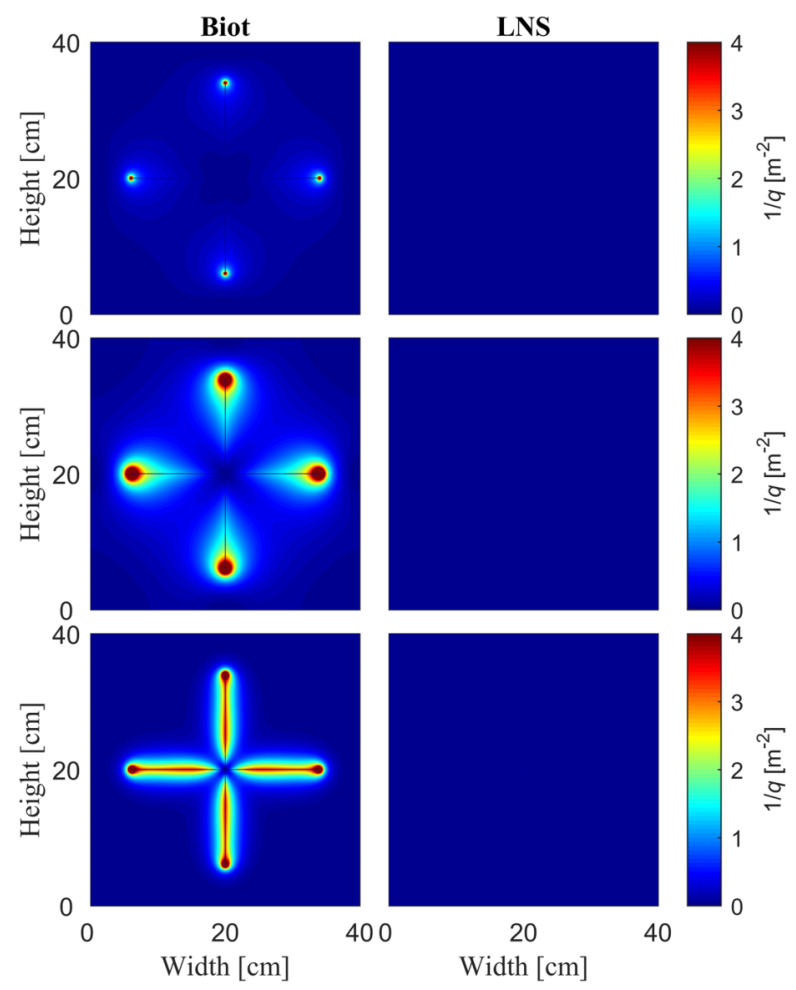

Figure 6 Local contribution $1 / q$ to the total attenuation $1 / Q$ in the spatial domain illustrated in Fig. 3, at (a) $f_{c 1} / 10$, (b) $f_{c 1}$ and (c) $f_{c 1}$ $\times 10$, where $f_{c 1}$ is the characteristic frequency of the low-frequency attenuation peak (Figs 4 and 5) associated with dissipation in the embedding background. One numerical solution is based on Biot's equations and the other on the coupled LNS equations. 


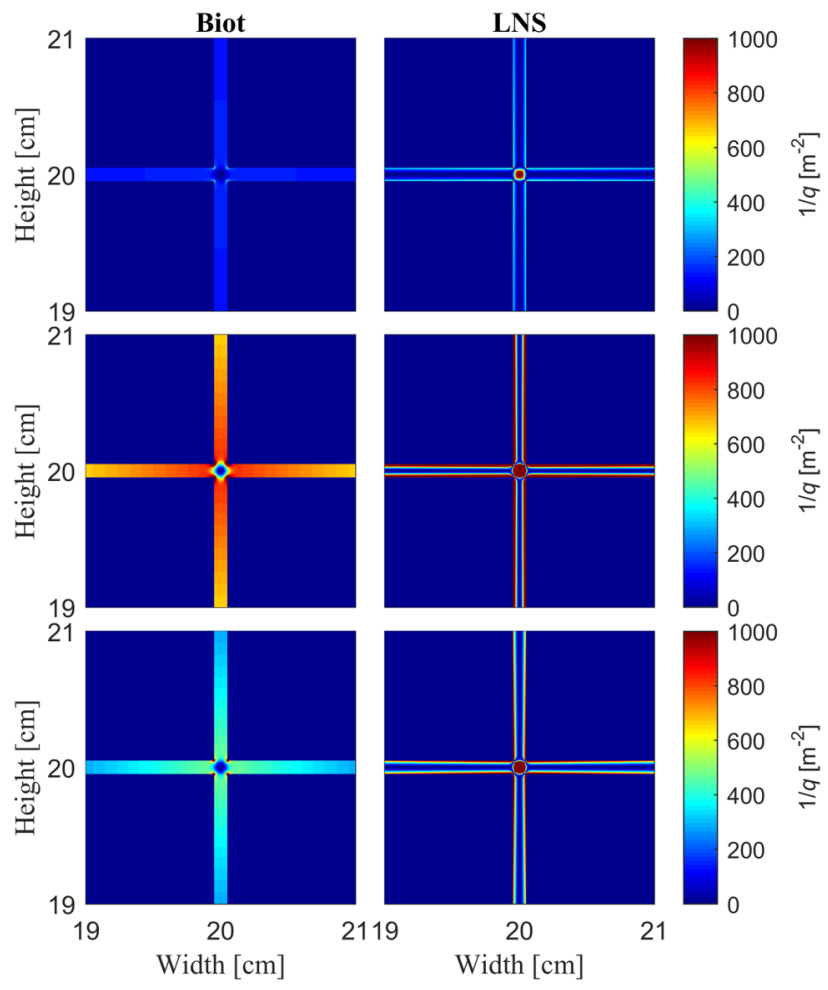

Figure 7 Zoom in on region A of the spatial domain illustrated in Fig. 3 for the local contribution $1 / q$ to the total attenuation $1 / Q$, at $f_{c 2} / 10$ (top), $f_{c 2}$ (middle) and $f_{c 2} \times 10$ (bottom), where $f_{c 2}$ is the characteristic frequency of the high-frequency attenuation peak (Figs 4 and 5) associated with squirt-flow dissipation within the cracks. At these high frequencies, the field $1 / q$ is not shown for the entire model because it is difficult to visualize the process within the very thin cracks. One numerical solution is based on Biot's equations and the other on the coupled LNS equations.

pressure difference between the differently oriented cracks is the cause of squirt flow. This high-frequency phenomenon is captured by both numerical schemes with excellent agreement between their results.

The employed numerical model as well as the material properties (Fig. 3; Tables 2 and 3) used for the results shown in Fig. 4 are identical to those used by Quintal et al. (2016) to solve the same set of coupled LNS equations and Biot's equations in the time domain. The main difference is that here, solving these equations in the frequency domain, we obtain results for a very broad frequency range comprising nine orders of magnitude $\left(10^{-2}\right.$ to $\left.10^{7} \mathrm{~Hz}\right)$, while the previous results covered only a range of four orders of magnitude $\left(10^{3}\right.$ to $10^{7} \mathrm{~Hz}$ ). In both cases, the frequency range could still be broadened, but this is significantly more difficult for simulations in the time domain because the time increment needs to
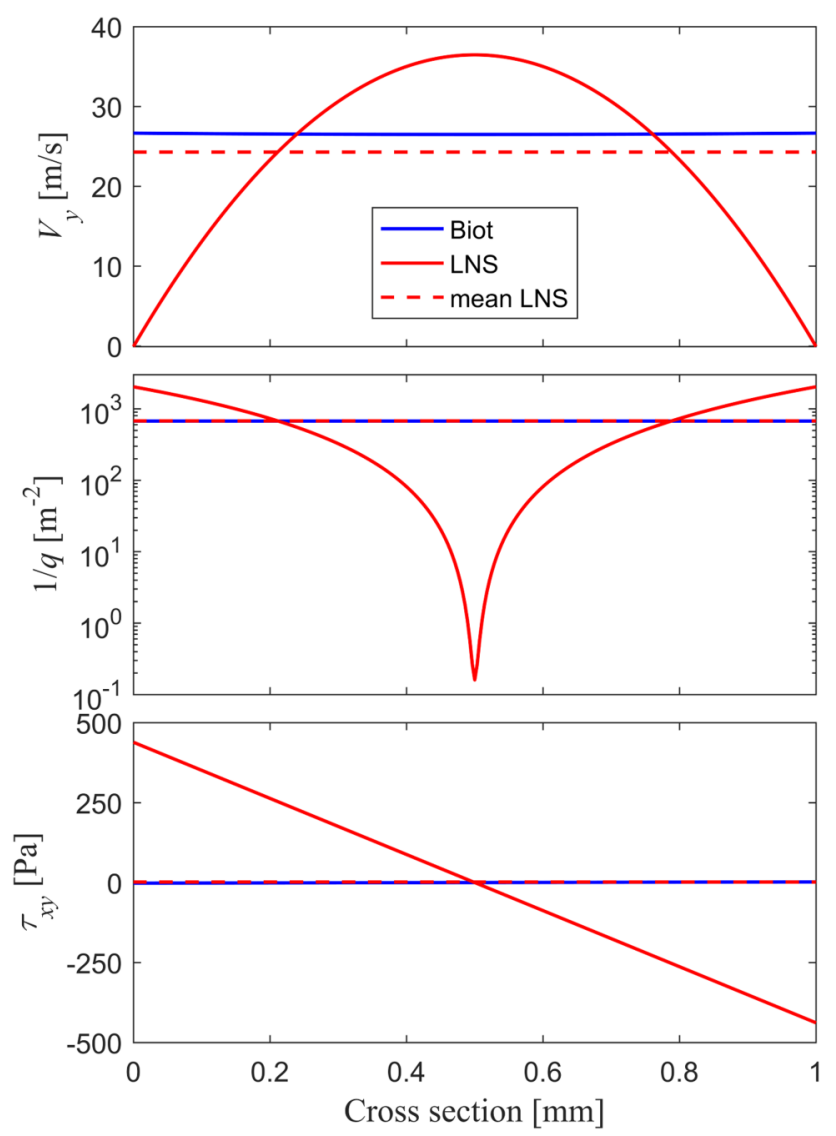

Figure 8 Fluid velocity in the vertical direction $V_{y}$, local contribution $1 / q$ to the total attenuation $1 / Q$ and shear stress in the fluid $\tau_{x y}$ on a cross-section of the vertical crack at $21 \mathrm{~cm}$ (Fig. 1) and at the characteristic frequency $f_{c 2}$ of the high-frequency attenuation peak (Figs 4, 5 and 7). One numerical solution is based on Biot's equations and the other on the coupled LNS equations. The dashed line indicates the mean value of the corresponding result based on the LNS solution.

be very small to accurately capture the physical phenomenon at high frequencies while the total time needs to be very long to also capture the physical phenomenon at low frequencies. This is why Quintal et al. (2016) only computed the dissipation associated with squirt flow. Furthermore, in comparison with their results based on Biot's equations in the time domain, our current results based on Biot's equations in the frequency domain are slightly different at frequencies between $10^{3}$ and $10^{4}$ $\mathrm{Hz}$, because only here the effect of the low-frequency FPD phenomenon, which is non-negligible at those frequencies, was accurately captured.

To verify that the results based on the coupled LNS equations (Fig. 4) correctly account for the total attenuation, we use the energy-based approach to compute the local attenuation contributions (equation (11)) of all elements and then 


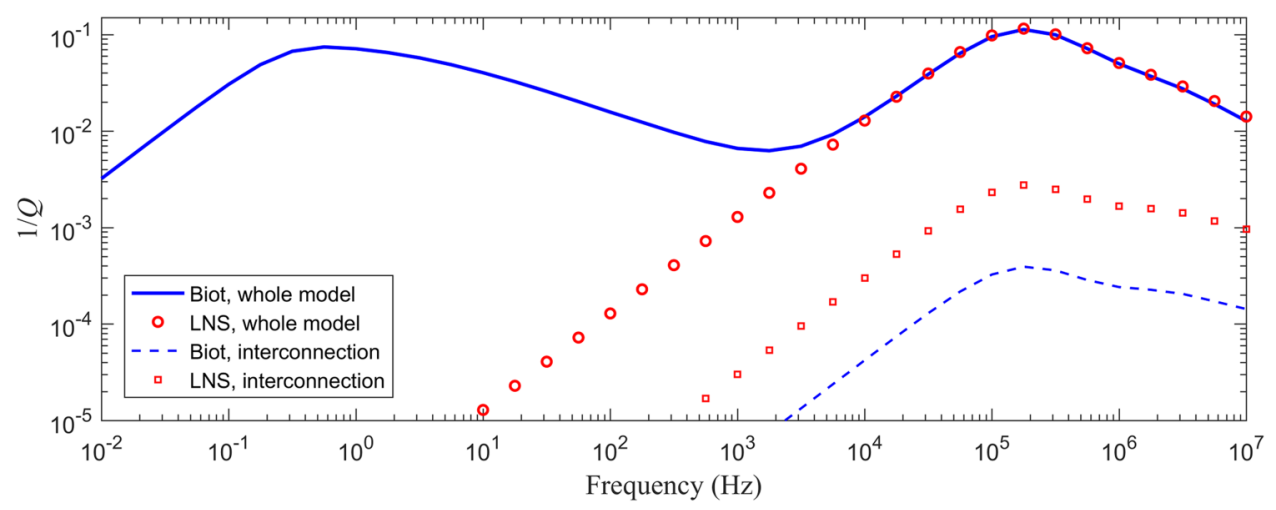

Figure 9 Attenuation 1/Q calculated using the energy approach in the square interconnection between cracks $\left(1 \mathrm{~mm}^{2}\right)$ and in the whole model (Fig. 3). These results are based on the numerical solutions of Biot's equations and the coupled LNS equations.

integrate them (equation (7)) to obtain the total attenuation. In Fig. 5, we observe excellent agreement between the results based on the energy-based approach and those obtained based on the viscoelastic assumption, which validates the use of the viscoelastic approach to upscale attenuation and dispersion characteristics from our heterogeneous medium described by the coupled LNS equations. For consistency, we do the same for the numerical scheme based on Biot's equations using the equations derived by Solazzi et al. (2016) and, as these authors point out, the agreement is nearly perfect (Fig. 5).

In addition to validating the viscoelastic assumption, the energy-based approach allows for quantifying the spatial distribution of the dissipation in the considered numerical model. Figure 6 displays the local attenuation field in the model shown in Fig. 3 at the characteristic frequency $f_{c 1}$ of the first attenuation peak (Figs 4 and 5), which occurs at lower frequencies and is associated with FPD in the background. Also shown are the corresponding results for two frequencies, which are higher and lower than $f_{c 1}$ by one order of magnitude, $f_{c 1} \times 10$ and $f_{c 1} / 10$. The results based on Biot's equations illustrate the spatial dissipation patterns in the low-porosity and low-permeability background. Conversely, as this phenomenon is not modelled by the scheme based on the coupled LNS equations, the corresponding images obviously show zero local attenuation throughout the model.

Figure 7 displays the local attenuation field at the characteristic frequency $f_{c 2}$ of the second attenuation peak (Figs 4 and 5), which occurs at higher frequencies and is due to squirt flow within the cracks. Also shown are the results for two frequencies, which are higher and lower than $f_{c 2}$ by one order of magnitude, $f_{c 2} \times 10$ and $f_{c 2} / 10$. These fields are shown only for a zoom in the centre of the model (area indicated by square $\mathrm{A}$ in Fig. 3), so that the squirt flow process within the thin cracks can be visualized. We see that local attenuation patterns based on Biot's equations and on the coupled LNS equations exhibit significant differences, despite the agreement of their results for the total attenuation shown in Fig. 4.

For a more detailed analysis, Fig. 8 depicts, at the characteristic frequency $f_{c 2}$, the fluid velocity in the vertical direction, the local attenuation and the shear stress in the fluid on a cross-section of the vertical crack at $21 \mathrm{~cm}$. For the results based on the coupled LNS equations, these three fields exhibit spatially variable profiles. The fluid velocity has a parabolic profile, the local dissipation exhibits a logarithmic decay towards the centre of the crack, and the linear shear stress is simply the gradient of the fluid velocity multiplied by the fluid viscosity. These variations of the fields across the crack occur because, when flowing, a viscous fluid resists the relative motion of virtual fluid layers with differing velocities, and this viscous resistance or friction causes dissipation. For the results based on Biot's equations, the corresponding profiles are approximately constant, in general appearing to be the mean value of the corresponding result observed for the coupled LNS equations. The profile of the local attenuation is, in fact, exactly the mean value of the variable one based on the coupled LNS equations, which demonstrates that the energy dissipation within the crack is correctly captured by the inherent upscaling associated with Biot's equations. On the other hand, looking again at Fig. 7, this does not seem to be the case in the small square region representing the interconnection between the two cracks. While the local dissipation computed with the coupled LNS equations is quite high in this region, low values are obtained with Biot's equations. We then quantify the contribution of the region representing the interconnection between the two cracks to the total attenuation and show the results from the two numerical schemes in Fig. 9. The dissipation accounted for by Biot's equations in this 
(a)
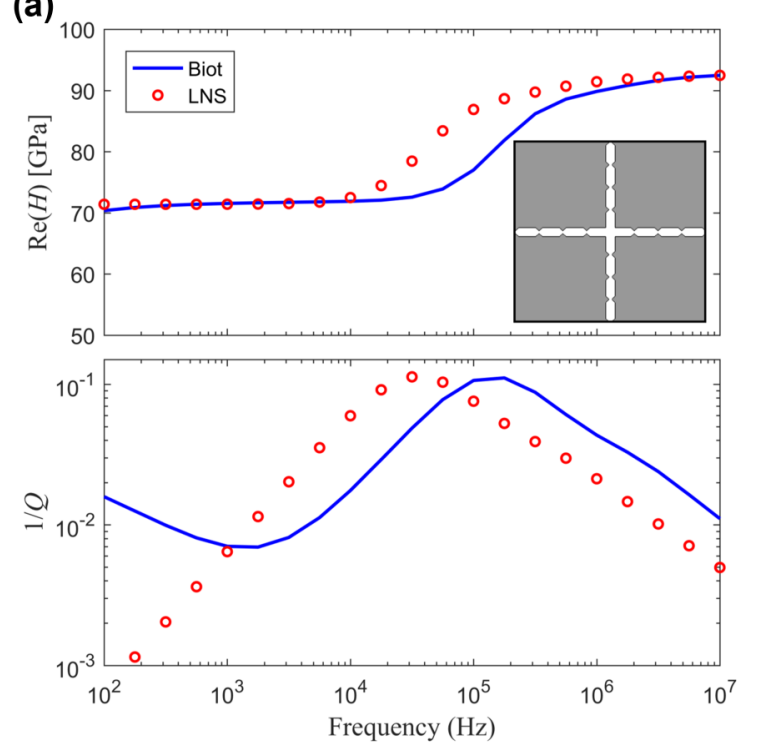

(b)

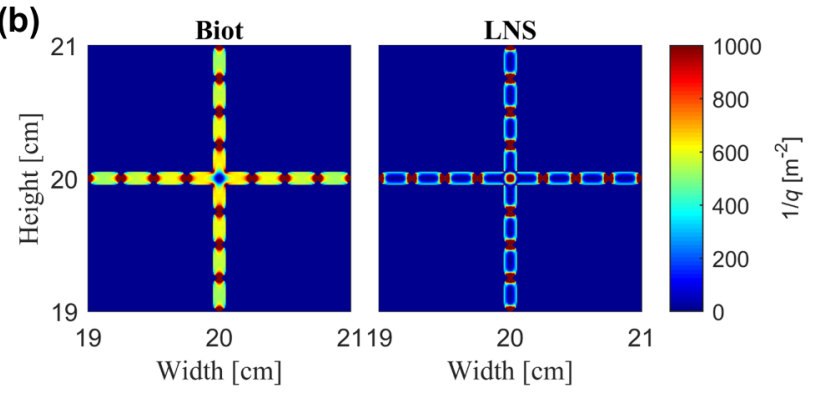

Figure 10 (a) Real part of the P-wave modulus $\operatorname{Re}(H)$ and attenuation $1 / Q$ calculated using the viscoelastic equivalent approach from the numerical solutions based on Biot's quasi-static equations and the coupled LNS equations. (b) Zoom in the central region of the spatial domain for the local contribution $1 / q$ to the total attenuation $1 / Q$ at $f_{c 2}$, where $f_{c 2}$ is the characteristic frequency of the attenuation peak associated with dissipation within the cracks.

region is indeed about one order of magnitude lower than that accounted for by the scheme based on the coupled LNS equations, revealing that Biot's equations fail in the interconnection between cracks, when applied to squirt flow scenarios that are essentially microscopic. The underestimation by Biot's equations of the dissipation in the interconnection between cracks is, however, negligible with respect to the total attenuation in the considered model.

\section{Interconnected cracks with asperities}

The discrepancy between results from the two numerical schemes in the interconnection between cracks could suggest a possible failure of Biot's equations in accounting for
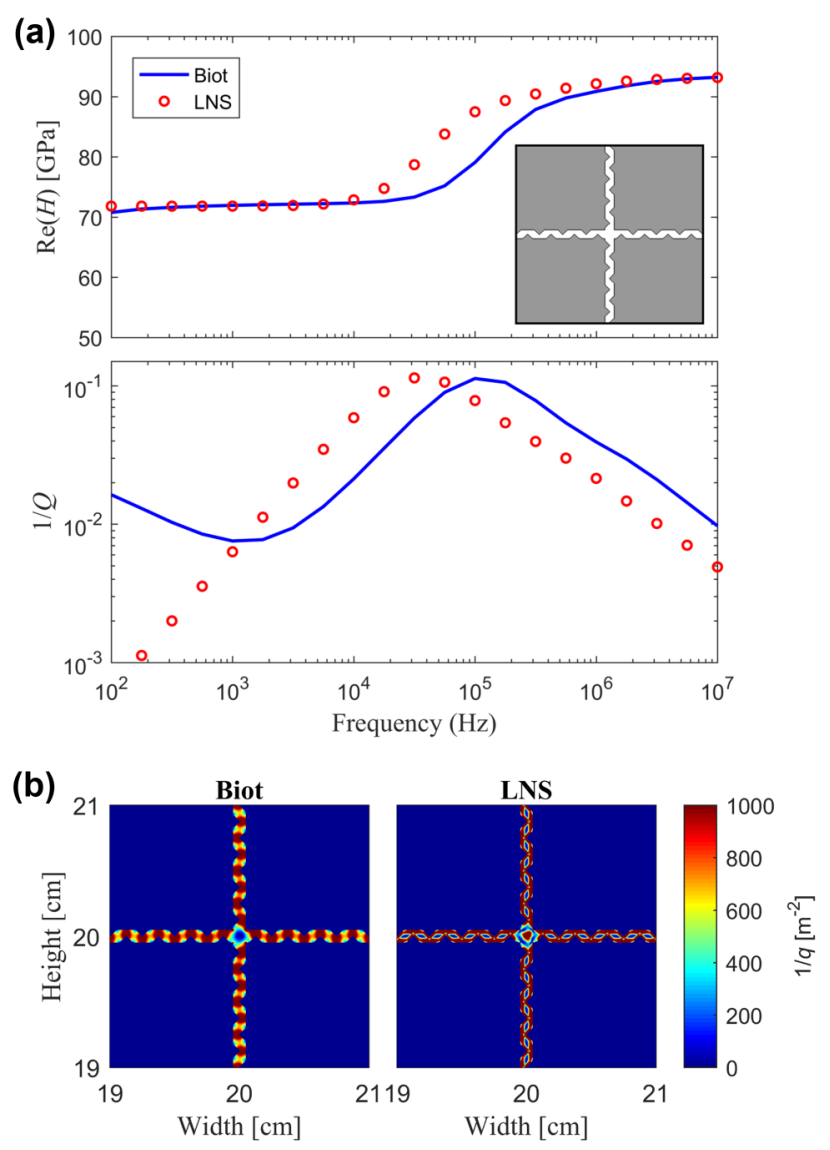

Figure 11 (a) Real part of the P-wave modulus $\operatorname{Re}(H)$ and attenuation $1 / Q$ calculated using the viscoelastic equivalent approach from the numerical solutions based on Biot's quasi-static equations and the coupled LNS equations. (b) Zoom in of the central region of the spatial domain for the local contribution $1 / q$ to the total attenuation $1 / Q$ at the characteristic frequency of the attenuation peak associated with dissipation within the cracks $f_{c 2}$.

the dissipation in, for instance, cracks having protuberant asperities on the walls. To investigate this, we consider two additional models of interconnected cracks, which are similar to those shown in Fig. 3 but with a significant amount of asperities of triangular shape along the crack walls. We use the same rock physical properties as for the previous model (Tables 2 and 3). First, we consider the asperities in phase making the opposite walls perfectly symmetric (inset in Fig. 10a). Second, we consider the asperities as being out of phase, which gives a tortuous character to the cracks (inset in Fig. 11b).

The results from the numerical simulations shown in Figs 10(a) and 11(a) indicate that Biot's equations account for the correct magnitude of dissipation in those models, although we observe a shift of the attenuation peak with respect 
(a)

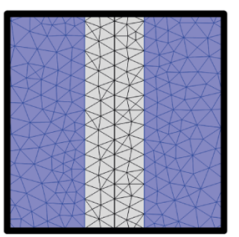

Fine

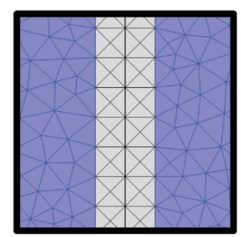

Medium

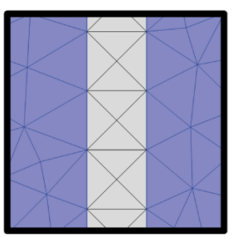

Coarse

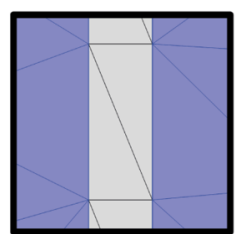

Very coarse
Figure 12 (a) Zoom in on numerical meshes with decreasing spatial resolution at an arbitrary part of a vertical crack (Fig. 3). (b) Corresponding numerical solutions based on Biot's equations and on the coupled LNS equations.

(b)
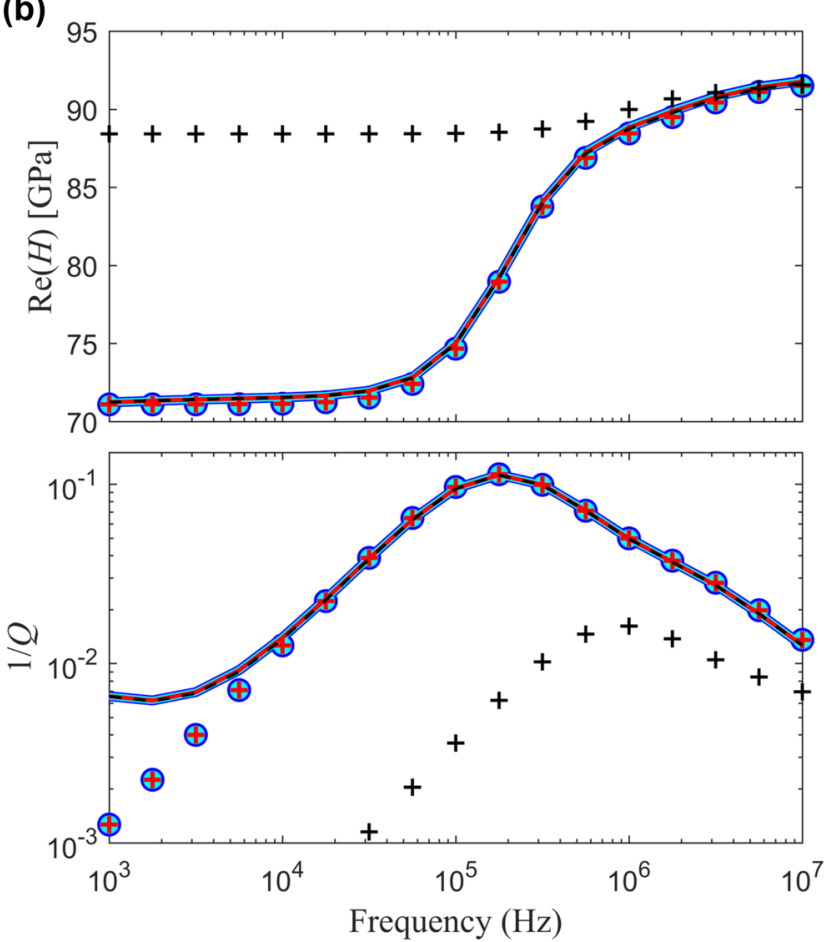

to frequency as compared with the results based on the coupled LNS equations. This shift is expected because the effective permeability within the cracks changes with the introduction of the asperities, while we still use the permeability obtained for the model shown in Fig. 3. To obtain good agreement of the numerical solutions with regard to the characteristic frequency would require estimating the permeability in the rough cracks for subsequent use in the numerical scheme based on Biot's equations.

We also inspect the local attenuation fields at the characteristic frequency $f_{c 2}$ of the squirt flow attenuation peak. Figure 10(b) reveals a certain similarity of the patterns obtained with the coupled LNS equations and with Biot's equations, with the highest values of attenuation occurring at the narrowest parts of the cracks. Figure 11(b) shows local attenuation patterns that are more complicated, but the highest values of attenuation occur also at the narrowest parts of the cracks, which are at the edges of the triangular asperities, for the results based on both numerical schemes.

In addition to the same magnitude for the total attenuation at the respective characteristic frequencies for the two numerical schemes (Figs 10a and 11a), just as we observed for the results from the model shown in Fig. 3, we observe in Figs 10(b) and 11(b), again, very low values in the local attenuation field at the crack intersection obtained with Biot's equations, in contrast to very high values obtained with the coupled LNS equations, with negligible effects for the total attenuation.

\section{Spatial resolution}

To assess the computational needs of the two numerical schemes in terms of spatial resolution inside the cracks, we 


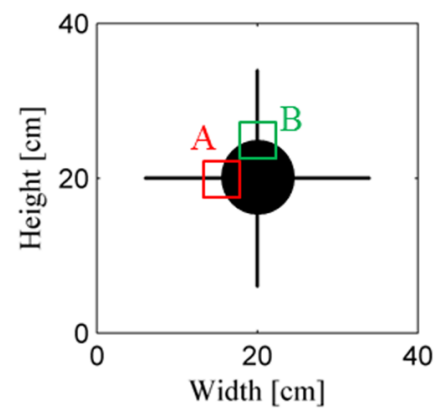

Figure 13 Sketch of the numerical model containing four rectangular cracks connected to a circular pore. To create this model, a circle with a radius of $4 \mathrm{~cm}$ was placed in the centre of the model shown in Fig. 3.

perform a series of simulations for the model shown in Fig. 3, with decreasing sizes of second-order (quadratic) numerical elements (Fig. 12a). Each triangular element has six nodal points distributed in the three vertices and in the middle of the three edges.

Figure 12(b) shows that, for the numerical solution based on the coupled LNS equations, the "coarse" mesh is adequate while the "very coarse" mesh provides incorrect results. For the numerical solution based on Biot's equations, however, the "very coarse" mesh still provides accurate results as the upscaled fields across the cracks, such as those observed in Figs 7 and 8, can be resolved by less nodal points than those based on the coupled LNS equations.

\section{Circular pore connected to rectangular cracks}

In this subsection, we modify the 2D numerical model shown in Fig. 3 by simply placing a circular void in its centre (Fig. 13). The new model can then be described as containing four compliant rectangular cracks, which could be interpreted as grain contacts at the microscopic scale, connected to a stiffer circular pore. We again perform numerical simulations based on both the coupled LNS equations and on Biot's equations. For consistency and to keep the analysis simple, the material properties used for the background and for the pore space are again those used for the model of interconnected cracks (Fig. 3; Tables 2 and 3). For the numerical scheme based on Biot's equations, the same material properties are used for the cracks and for the circular pore (Table 1).

Figure 14 shows the numerical results based on the viscoelastic equivalent approach for the model shown in Fig. 13. Two attenuation peaks accompanied by two corresponding inflections of the dispersion curves are obtained with Biot's equations. The attenuation peak and corresponding dispersion at lower frequencies are caused by FPD in the background. The attenuation peak and corresponding dispersion at higher frequencies are caused by squirt flow. We again observe excellent agreement between the results from both numerical schemes for the squirt flow mechanism. This shows that Biot's equations are suitable also for microscopic scenarios where a solid frame present within in the circular pore is rather unrealistic as well as the relatively high permeability

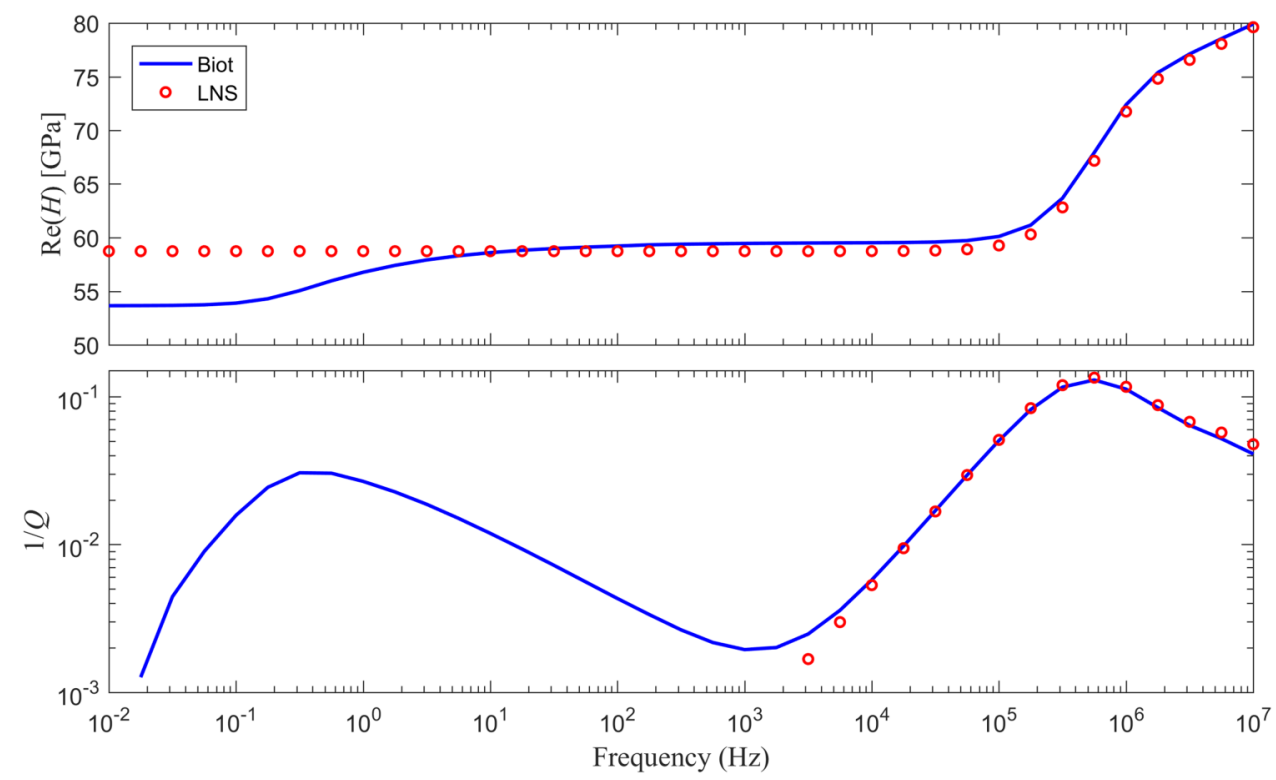

Figure 14 Real part of the P-wave modulus $\operatorname{Re}(H)$ and attenuation $1 / Q$ calculated, based on the viscoelastic equivalent approach, from numerical solutions of Biot's quasi-static equations and the coupled LNS equations. 

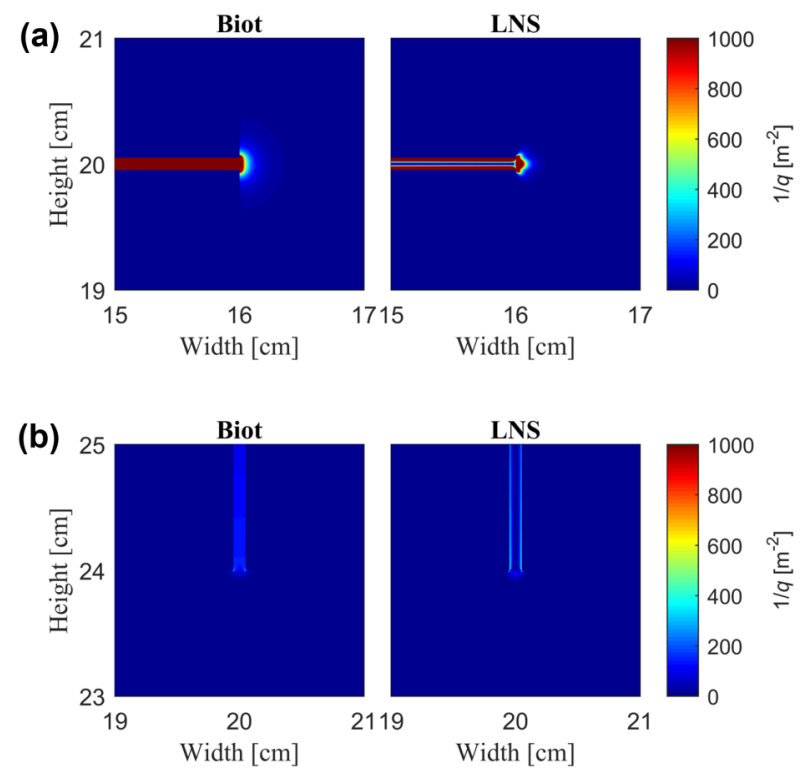

Figure 15 Zoom in of regions (a) A and (b) B indicated in Fig. 13 showing the local contribution $1 / q$ to the total attenuation $1 / Q$ at the characteristic frequency $f_{c 2}$ of the attenuation peak (high-frequency peak in Fig. 14) associated with dissipation within the cracks.

and porosity existing in the background medium, as compared with those of solid grains.

To better understand the squirt flow phenomenon prevailing in the considered model, it is useful to remember that, in the compression cycle of the oscillatory test, the applied vertical compression deforms significantly only the horizontal cracks. This causes fluid to flow, or FPD, from the horizontal cracks into the circular pore and then, to a much lesser extent, from the circular pore into the vertical cracks. Correspondingly, higher dissipation occurs in the horizontal cracks, as illustrated by the local attenuation fields shown in Fig. 15.

\section{Comparison between two squirt flow scenarios}

It is interesting to compare the dissipation processes prevailing in the two different models shown in Figs 3 and 13. On the one hand, we have just illustrated in Fig. 15 that, when the four cracks are connected to a circular pore (Fig. 13) and compression is vertical, most of the dissipation occurs in the horizontal cracks. On the other hand, Fig. 7 shows that, when cracks are connected directly to each other (Fig. 3), dissipation of similar magnitude occurs in both horizontal and vertical cracks. This difference in the dissipation processes of the two models is quantified in Fig. 16. For directly interconnected cracks, both horizontal and vertical cracks contribute similarly to the total attenuation in the model, while for cracks connected to a
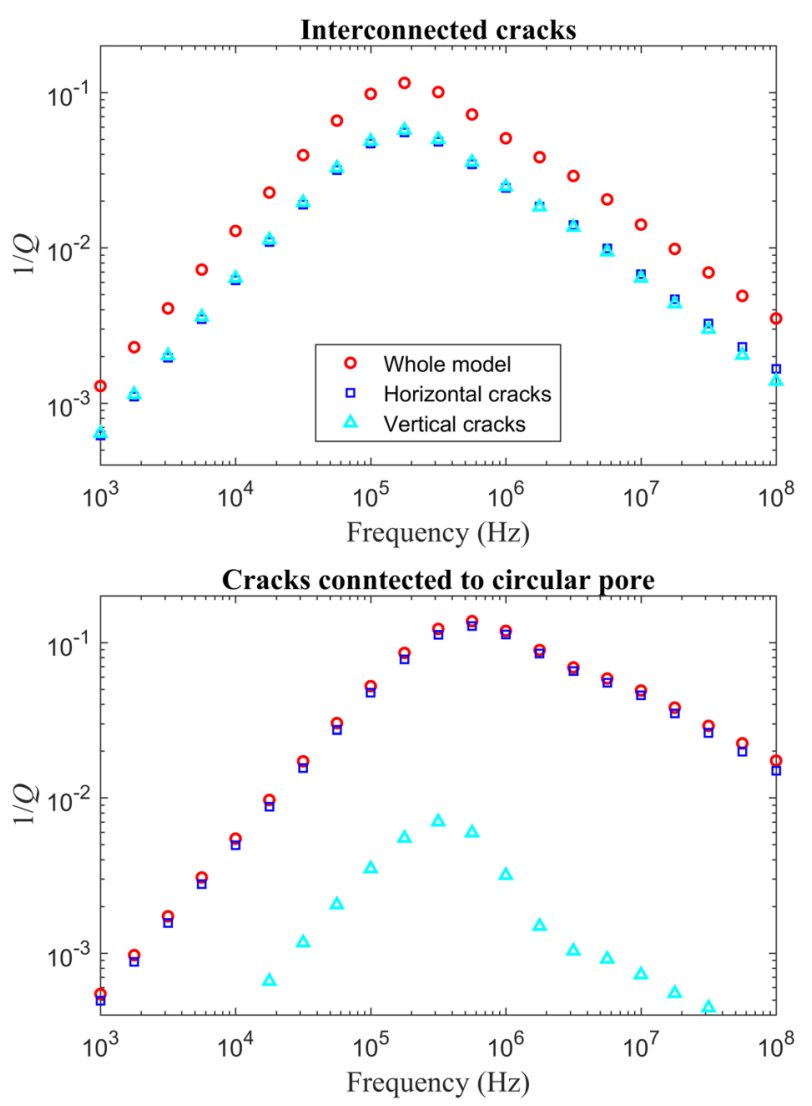

Figure 16 Attenuation $1 / Q$ calculated using the energy approach from the numerical scheme based on the coupled LNS equations for the models shown in Fig. 3 (upper plot) and Fig. 13 (lower plot). The attenuation contributions are from (i) the whole model, (ii) from horizontal cracks only and (iii) from vertical cracks only.

circular pore, the horizontal cracks account for almost the entire dissipation, considering vertical compression.

\section{Spatial scale}

The 2D squirt flow models discussed here were presented with centimetric dimensions (Figs 3 and 13). This is clearly disturbing, particularly for the model shown in Fig. 13, which resembles a typical microscopic squirt flow scenario. It is thus important to remark that the absolute spatial dimensions of those models have no effect on attenuation and dispersion caused by squirt flow. In terms of geometry, squirt flow depends only on dimensionless attributes. For example, the characteristic frequency is controlled by the aspect ratio of the cracks (ratio of aperture to length) having smooth walls, as predicted by corresponding analytical studies (O'Connell and Budiansky 1977; Murphy et al. 1986; Gurevich et al. 2010). 
This is corroborated by the fact that, when the models shown in Figs 3 and 13 were rescaled, the simulations produced results for squirt flow that are identical to those shown in Figs 4 and 14 . This was done by rescaling the $40 \times 40-\mathrm{cm}$ models into $400 \times 400-\mu \mathrm{m}$ models while keeping the relative dimensions unchanged.

\section{CONCLUSIONS}

We extended and complemented a previous study by modifying its numerical scheme based on a solution of the coupled Lamé-Navier and Navier-Stokes (LNS) equations to simulate, here in the frequency domain, squirt flow in cracks embedded in an elastic solid. Relying on the assumption that the corresponding heterogeneous models behave effectively as viscoelastic homogeneous media at a larger scale, the resulting attenuation and stiffness modulus dispersion were based on volume averages of the stress and strain fields over the entire computational domain. To test this assumption, we implemented an energy-based approach to compute the local attenuation based on the dissipated and stored energy in each element of the numerical model. Integration of the local attenuation over the entire model yielded results which precisely agree with the attenuation results from stress-strain volume averages based on, and thus validating, the viscoelastic assumption. Additionally, the energy-based approach provides an accurate means to locally study the dissipation phenomenon by allowing for a visualization of the heterogeneous dissipation field within the model and a quantification of the contribution from certain subdomains to the total attenuation.

We performed 3D simulations based on the coupled LNS equations considering a squirt flow model that consisted of a spherical crack embedded in an elastic solid. The characteristic frequency observed in the numerical results was compared with that of a corresponding analytical solution. Their good agreement indicates that the dissipative viscous flow problem is correctly handled in our numerical solution of the linearized, quasi-static Navier-Stokes equations. These numerical results were also compared with those based on a 2D model to allow for a first-order assessment of 3D effects that are neglected in 2D studies. Our 2D study overestimates attenuation.

We performed 2D simulations based on the coupled LNS equations considering two squirt flow models. One model consisted of interconnected cracks and the other one of cracks connected to a circular pore. For comparison, we also performed corresponding simulations based on Biot's equations. We observed that the fluid velocity profile across a crack aperture is parabolic when computed with the linearized Navier-Stokes equations, while it is approximately constant when computed with Biot's equations. Meanwhile, the local attenuation based on the Navier-Stokes equations exhibit a logarithmic decay towards the centre of the crack aperture, while the one based on Biot's equations is constant and equal to the mean of the variable value based on the Navier-Stokes equations. Despite these differences in the fluid velocity and local attenuation fields, the results from the different numerical schemes exhibited excellent agreement for the total P-wave attenuation and modulus dispersion caused by squirt flow. A detailed inspection of the local attenuation fields, however, indicates that Biot's equations fail to account for the correct amount of dissipation in the interconnection between cracks, in comparison with the results based on the coupled LNS equations.

Additional 2D simulations considering interconnected cracks with asperities along their walls revealed that the magnitude of the total attenuation is accurately quantified by Biot's equations for these models as well. The only difference with respect to the results from the coupled LNS equations was a frequency shift of the resulting attenuation curves. This shift occurs because the effective permeability in the cracks changes with the insertion of asperities, which was naturally accounted for by the scheme based on the coupled LNS equations, but not for Biot's equations. While corresponding effective models for permeability as well as for stiffness are available, the fact that the numerical scheme based on the coupled LNS equations does not require any effective crack/pore properties makes it highly suitable for realistic simulations of squirt flow effects in complex models, such as those derived from high-resolution microtomographic images of rock samples. On the other hand, compared with Biot's equations, the scheme based on the coupled LNS equations does require a larger spatial resolution and thus more computational power to simulate the variable fluid velocity and local attenuation profiles within the cracks. To alleviate this problem, we used a quadratic finite-element formulation, which allowed for approximating the quadratic fluid velocity profile in the cracks with a relatively low number of elements across their aperture.

\section{ACKNOWLEDGEMENTS}

Beatriz Quintal acknowledges the funding from the Swiss $\mathrm{Na}$ tional Science Foundation (SNF) through the project 172691 and Holger Steeb acknowledges the funding from the German Science Foundation (DFG) through the project STE-969-13/1. Additionally, this work has been completed within the Swiss 
Competence Center on Energy Research - Supply of Electricity with the support of the Innosuissse. We thank Jürg Hunziker and Germán Rubino for helpful comments and insightful discussions.

\section{DATA AVAILABILITY STATEMENT}

The numerical results presented here are reproducible by solving the same equations under equivalent boundary conditions, but they can be shared by contacting the first author.

\section{ORCID}

Beatriz Quintal (D) https://orcid.org/0000-0001-6714-420X

Klaus Holliger (D) https://orcid.org/0000-0002-2584-8177

Holger Steeb (D) https://orcid.org/0000-0001-7602-4920

\section{REFERENCES}

Adelinet M., Fortin J., Guéguen Y., Schubnel A. and Geoffroy L. 2010. Frequency and fluid effects on elastic properties of basalt: experimental investigations. Geophysical Research Letters 37, L02303.

Batzle M.L., Han D.-H. and Hofmann R. 2006. Fluid mobility and frequency-dependent seismic velocity - Direct measurements. Geophysics 71, N1-N9.

Berryman J.G. 2005. Comparison of upscaling methods in poroelasticity and its generalizations. Journal of Engineering Mechanics 131, 928-936.

Best A.I., McCann C. and Sothcott J. 1994. The relationships between the velocities, attenuations and petrophysical properties of reservoir sedimentary rocks. Geophysical Prospecting 42, 151-178.

Biot M.A. 1941. General theory of three-dimensional consolidation. Journal of Applied Physics 12, 155-164.

Biot M.A. 1962. Mechanics of deformation and acoustic propagation in porous media. Journal of Applied Physics 33, 1482-1498.

Brajanovski M., Gurevich B. and Schoenberg M. 2005. A model for P-wave attenuation and dispersion in a porous medium permeated by aligned fractures. Geophysical Journal International 163, 372384.

Burridge R. and Keller J.B. 1981. Poroelasticity equations derived from microstructure. Journal of the Acoustical Society of America 70, 1140-1146.

Carcione J.M., Santos J.E. and Picotti S. 2011. Anisotropic poroelasticity and wave-induced fluid flow: harmonic finite-element simulations. Geophysical Journal International 186, 1245-1254.

Chapman M., Zatsepin S.V. and Crampin S. 2002. Derivation of a microstructural poroelastic model. Geophysical Journal International 151, 427-451.

Chapman S., Borgomano J., Yin H., Fortin J. and Quintal B. 2019. Forced oscillation measurements of seismic wave attenuation and stiffness moduli dispersion in glycerine-saturated Berea sandstone. Geophysical Prospecting 67, 956-968.
Chapman S., Tisato N., Quintal B. and Holliger K. 2016. Seismic attenuation in partially saturated Berea sandstone submitted to a range of confining pressures. Journal of Geophysical Research Solid Earth 121, 1664-1676.

Collet O. and Gurevich B. 2016. Frequency dependence of anisotropy in fluid saturated rocks - Part I: aligned cracks case. Geophysical Prospecting 64, 1067-1084.

Dvorkin J., Mavko G. and Nur A. 1995. Squirt flow in fully saturated rocks. Geophysics 60, 97-107.

Gassmann F. 1951. Über die Elastizität poröser Medien. Vierteljahrsschrift der Naturforschenden Gesellschaft in Zürich 96, 1-23.

Gurevich B., Makarynska D., de Paula O.B. and Pervukhina M. 2010. A simple model for squirt-flow dispersion and attenuation in fluidsaturated granular rocks. Geophysics 75, N109-N120.

Hunziker J., Favino M., Caspari E., Quintal B., Rubino J.G., Krause R. et al. 2018. Seismic attenuation and stiffness modulus dispersion in porous rocks containing stochastic fracture networks. Journal of Geophysical Research: Solid Earth 123, 125-143.

Jänicke R., Quintal B. and Steeb H. 2015. Numerical homogenization of mesoscopic loss in poroelastic media. European Journal of Mechanics A/Solids 49, 382-395.

Jaeger J.C., Cook N.G.W. and Zimmerman R.W. 2007. Fundamentals of Rock Mechanics, 4th ed. Blackwell Publishing.

Landau L.D. and Lifshitz E.M. 1959. Fluid Mechanics, 2nd ed. Pergamon Press.

Madonna C., Quintal B., Frehner M., Almqvist B.S.G., Tisato N., Pistone M. et al. 2013. Synchrotron-based X-ray tomographic microscopy for rock physics investigations. Geophysics 78, D53D64.

Madonna C., Almqvist B.S.G. and Saenger E.H. 2012. Digital rock physics: numerical prediction of pressure-dependent ultrasonic velocities using micro-CT imaging. Geophysical Journal International 189, 1475-1482.

Masson Y.J. and Pride S.R. 2007. Poroelastic finite-difference modeling of seismic attenuation and dispersion due to mesoscopic-scale heterogeneity. Journal of Geophysical Research 112, B03204.

Mavko G. and Jizba D. 1991. Estimating grain-scale fluid effects on velocity dispersion in rocks. Geophysics 56, 1940-1949.

Milani M., Rubino J.G., Müller T.M., Quintal B., Caspari E. and Holliger K. 2016. Representative elementary volumes for evaluating effective seismic properties of heterogeneous poroelastic media. Geophysics 81, D169-D181.

Murphy W.F., Winkler K.W. and Kleinberg R.L. 1986. Acoustic relaxation in sedimentary rocks, dependence on grain contacts and fluid saturation. Geophysics 51, 757-766.

Nakagawa S. and Schoenberg M. 2007. Poroelastic modeling of seismic boundary conditions across a fracture. Journal of the Acoustical Society of America 122, 831-847.

O’Connell R.J. and Budiansky B. 1977. Viscoelastic properties of fluid-saturated cracked solids. Journal of Geophysical Research: Solid Earth 82, 5719-5735.

O'Connell R.J. and Budianski B. 1978. Measures of dissipation in viscoelastic media. Geophysical Research Letters 5, 5-8.

Pimienta L., Fortin J. and Guéguen Y. 2015. Bulk modulus dispersion and attenuation in sandstones. Geophysics 80, D111D127. 
Plawsky J.L. 2014. Transport Phenomena Fundamentals. CRC Press. Pride S.R., Berryman J.G. and Harris J.M. 2004. Seismic attenuation due to wave-induced flow. Journal of Geophysical Research 109, B01201.

Quintal B., Jänicke R., Rubino J.G., Steeb H. and Holliger K. 2014. Sensitivity of S-wave attenuation to the connectivity of fractures in fluid-saturated rocks. Geophysics 79, WB15-WB24.

Quintal B., Rubino J.G., Caspari E. and Holliger K. 2016. A simple hydromechanical approach for simulating squirt-type flow. Geophysics 81, D335-D344.

Quintal B., Steeb H., Frehner M. and Schmalholz S.M. 2011. Quasistatic finite-element modeling of seismic attenuation and dispersion due to wave-induced fluid flow in poroelastic media. Journal of Geophysical Research: Solid Earth 116, B01201.

Rubino J.G., Guarracino L., Müller T.M. and Holliger K. 2013. Do seismic waves sense fracture connectivity? Geophysical Research Letters 40, 50127.

Rubino J.G., Ravazzoli C.L. and Santos J.E. 2009. Equivalent viscoelastic solids for heterogeneous fluid-saturated porous rocks. Geophysics 74, N1-N13.

Sams M.S., Neep J.P., Worthington M.H. and King M.S. 1997. The measurement of velocity dispersion and frequency-dependent intrinsic attenuation in sedimentary rocks. Geophysics 62, 14561464.

Shewchuk J.R. 2002. Delaunay refinement algorithms for triangular mesh generation. Computational Geometry 22, 21-74.

Solazzi S.G., Rubino J.G., Müller T.M., Milani M., Guarrancino L. and Holliger K. 2016. An energy-based approach to estimate seismic attenuation due to wave-induced fluid flow in heterogeneous poroelastic media. Geophysical Journal International 207, 232823.
Subramaniyan S., Quintal B., Madonna C. and Saenger E.H. 2015. Laboratory-based seismic attenuation in Fontainebleau sandstone: evidence of squirt flow. Journal of Geophysical Research: Solid Earth 120, 7526-7535.

Tisato N. and Quintal B. 2013. Measurements of seismic attenuation and transient fluid pressure in partially saturated Berea sandstone: evidence of fluid flow on the mesoscopic scale. Geophysical Journal International 195, 342-351.

Vinci C., Renner J. and Steeb H. 2014. On attenuation of seismic waves associated with flow in fractures. Geophysical Research Letters 41, 7515-7523.

Wenzlau F., Altmann J. and Müller T.M. 2010. Anisotropic dispersion and attenuation due to wave-induced fluid flow: quasi-static finite-element modeling in poroelastic solids. Journal of Geophysical Research: Solid Earth 115, B07204.

Whitaker S. 1986. Flow in porous media I: a theoretical derivation of Darcy's law. Transport in Porous Media 1, 3-25.

White J.E. 1975. Computed seismic speeds and attenuation in rocks with partial gas saturation. Geophysics 40, 224-232.

White J.E., Mikhaylova N.G. and Lyakhovitskiy F.M. 1975. Lowfrequency seismic waves in fluid-saturated layered rocks: izvestiya, academy of sciences. USSR. Physics of the Solid Earth 11, 654-659. Winter H.H. 1987. Viscous dissipation term in energy equations. In: Modular Instruction Series C: Calculation and Measurement Techniques for Momentum, Energy and Mass Transfer, Vol. 7 (ed. J.R. Gordon), pp. 27-34. American Institute of Chemical Engineers, New York.

Zhang Y. and Toksöz M.N. 2012. Computation of dynamic seismic responses to viscous fluid of digitized three-dimensional Berea sandstones with a coupled finite-difference method. Journal of the Acoustical Society of America 132, 630-640. 